Bioelectrochemistry and Bioenergetics, 25 (1991) 395-413

A section of J. Electroanal. Chem., and constituting Vol. 320 (1991)

Elsevier Sequoia S.A., Lausanne

\title{
Dual-frequency excitation: a novel method for probing the nonlinear dielectric properties of biological systems, and its application to suspensions of $S$. cerevisiae
}

\author{
Andrew M. Woodward and Douglas B. Kell * \\ Department of Biological Sciences, University College of Wales, Aberystwyth, Dyfed SY23 3DA (UK)
}

(Received 30 November 1990)

\begin{abstract}
We have recently described the construction of a dual-cell, nonlinear dielectric spectrometer, and its application to the study of cell suspensions of $S$. cerevisiae (A.M. Woodward and D.B. Kell, Bioelectrochem. Bioenerg., 24 (1990) 83). Substantial, odd harmonics were generated by these cells when stimulated by very modest sinusoidal electrical fields, within fairly sharp voltage- and frequency windows (ca. $0.8-2.5 \mathrm{~V} \mathrm{~cm}^{-1}, 1-50 \mathrm{~Hz}$ ). Resting cells were found to generate only odd-numbered harmonics. In the present work, we have simultaneously applied two sinusoidal frequencies which were indinidually of unsuitable frequency and/or amplitude for the generation of harmonics when applied to suspensions of S. cerevisiae. Strong "sidebands" or "beat frequencies" were observed which were the (odd-numbered) sums and differences of the exciting frequencies (viz. $f_{1} \pm 2 f_{2}, f_{2} \pm 2 f_{1}$ ). The generation of these beat frequencies was strongly inhibited by low concentrations of sodium metavanadate, suggesting that they may be ascribed largely to the $\mathrm{H}^{+}$-ATPase present in the plasma membranes of these cells. We show that the ability of dc fields to inhibit the manifestation of nonlinear dielectric behaviour by these cells is explicable in terms of their ability to act as a field of zero $\mathrm{Hz}$, forcing the excitation out of the amplitude window. When the cells were allowed to glycolyse, beat frequencies of even order $\left(f_{1} \pm f_{2}, f_{1} \pm 3 f_{2}\right)$ were observed. The present approach provides a novel and powerful approach to the registration of nonlinear dielectric spectra, which, due to the greater precision inherent in the discrimination of frequencies rather than voltages may be expected to provide a more sensitive means of detecting nonlinear dielectric properties in biological systems. If the transduction of exogenous electrical field energy recorded by this method is representative of the natural turnover of the $\mathrm{H}^{+}$-ATPase in vivo, it may be calculated that the efficiency of the capture of electric field energy by this enzyme is some $3 \%$.
\end{abstract}

\section{INTRODUCTION}

We have recently described the construction of a dual-cell, software-based nonlinear dielectric spectrometer, and its application to the study of cell suspensions

\footnotetext{
ऋ To whom correspondence should be addressed.
}

0302-4598/91/\$03.50 C $1991-$ Elsevier Sequoia S.A. 
of $S$. cerevisiae [1]. Substantial, odd harmonics were generated by these cells when stimulated by very modest sinusoidal electrical fields, within fairly sharp voltageand frequency windows (ca. $0.8-2.5 \mathrm{~V} \mathrm{~cm}^{-1}, 2-50 \mathrm{~Hz}$ ). Resting cells were found to generate only odd- numbered harmonics, and inhibitor studies indicated that the great majority of the signal could be ascribed to the membrane $\mathrm{H}^{+}$-ATPase possessed by these organisms.

The basis for the generation of these nonlinear dielectric spectra lies in the fact that enzymes are not dipolar billiard balls but pass through conformations, each with different permanent and induced dipole moments, in a sequential or hierarchical fashion, with some or all of the steps being coupled to observable catalytic activity [2-6]. The corollary of this is that such enzymes should indeed possesss unusual and nonlinear dielectric spectra [7-9], as was observed [1]. Such behaviour may also account for the failure of superpositions of Debye-like relaxations to account for the dielectric behaviour of biological systems and other slowly relaxing, strongly interacting materials [10-13].

Because of the fact that there are many reaction intermediates in this enzyme, whose individual kinetic properties are understood only in part [14,15], the optimum stimulating frequency for the generation of harmonics undoubtedly represents a "compromise" between the individual rate constants of the steps contributing to the overall turnover of the enzyme.

Another property of nonlinear systems (additional to their ability to generate harmonics when stimulated by a pure sinusoid) is that if they are excited by stimuli at more than one frequency they will tend to generate frequencies that are not harmonics but are sums and differences of (integral multiples of) the exciting frequencies ([16-18] and see below). Since in principle one could excite the system with individual frequencies that are closer to the inverse rate-constants of the individual steps, this property might be expected to provide even more kinetic information than that to be obtained from the nonlinear dielectric approach based on the production of harmonics alone. The purpose of the present work was therefore to study the response of the nonlinear yeast ATPase system characterised previously [1], when stimulated by electrical fields containing more than one sinusoidal frequency. A preliminary account of this work has been presented [19].

\section{THEORY}

A generalised centrosymmetric system is most easily visualised using the "ball bearing in a bowl" analogy, in which we consider the horizontal displacement $x(t)$ of the ball bearing when released from a point on the surface of the bowl. If only even- power terms are present in the potential (e.g., for a parabola where terms above the squared term are absent):

$\phi=a+b x(t)^{2}+\ldots$

these terms are symmetric about the origin and form a symmetric bowl. Thus the 
ball bearing will be forced into a symmetric motion about the origin, since only odd terms can be present in its equation of motion:

$\mathrm{d}^{2} x(t) / \mathrm{d} t^{2} \propto \mathrm{d} \phi(x) / \mathrm{d} x(t)=d x(t)+e x(t)^{3}+\ldots$

Consequently, when the driving term for the equation of motion is sinusoidal, only odd-order powers of this sinusuid are produced in the displacement:

$x(t)=k(\omega) \cos (\omega t)+l(\omega) \cos ^{3}(\omega t)+\ldots$

Trigonometric manipulation of these into a harmonic expansion produces only odd-numbered harmonics of the driving frequency:

$x(t)=p(\omega) \cos (\omega t)+q(\omega) \cos (3 \omega t)+\ldots$

The introduction of odd-order terms into the power series expansion of the potential effectively "skews" the bowl and breaks the symmetry of oscillation. Consequently even-order terms appear in the equation of motion, giving rise to even-numbered harmonics when the system in excited by a sinusoidal field.

The membrane $\mathrm{H}^{+}$-ATPase of resting yeast cell suspensions at static head comprises a centrosymmetric system, as can be seen from the generation of only odd harmonics [1].

This means that in the power series expansion of the dielectric polarisation, $P$, caused by the imposition of an electromagnetic field,

$P=P_{1}+P_{2}+P_{3}+\ldots$

only the odd order terms are present $[17,18]$, and since the system is weakly nonlinear, then:

$P_{1} \gg P_{3} \gg P_{5}$

so that

$P=P_{1}+P_{3}+0\left(P_{5}\right)$

A sinusoidal input signal

$E=E_{0} \sin (\omega t+\theta)$

gives the linear term

$P_{1}=\chi^{(1)}(\omega) E$

and the 3rd-order coupling term

$P_{3}=\chi^{(3)}(3 \omega) E^{3}$

where $\chi^{(n)}(\omega)$ is the $n$th order dielectric susceptibility evaluated at $\omega[17,18]$.

We next generalise the second-order frequency-mixing analysis of [17] to higher order using the above considerations. If a twin-frequency signal

$E(t)=E_{1}+E_{2}$

where

$E_{1}=E_{0,1} \sin \left(\omega_{1} t+\theta_{1}\right)$ 
and

$E_{2}=E_{0,2} \sin \left(\omega_{2} t+\theta_{2}\right)$

is applied, then the linear term is

$P_{1}=\chi^{(1)}\left(\omega_{1}\right) E_{1}+\chi^{(1)}\left(\omega_{2}\right) E_{2}$

The $n$ th-order coupling term is formed from the sum of all possible combinations of $m$ individual inputs which can give rise to $n$ th-order products, since it originates from the binomial expansion of a term in the polarization expansion of the form $\chi^{(n)}\left(E_{1}+E_{2}+\ldots+E_{m}\right)^{n}$, and arises physically from the nonlinear response to the beating between the individual input frequencies [17]. Thus the 3 rd-order coupling term is of the form $\chi^{(3)}\left(E_{1}+E_{2}\right)^{3}$, i.e.

$$
\begin{aligned}
P_{3}= & \chi^{(3)}\left(3 \omega_{1}\right) E_{1}^{3}+\chi^{(3)}\left(3 \omega_{2}\right) E_{2}^{3}+\chi^{(3)}\left(\omega_{1}+2 \omega_{2}\right) E_{1} E_{2}^{2}+\chi^{(3)}\left(\omega_{1}-2 \omega_{2}\right) E_{1} E_{2}^{2} \\
& +\chi^{(3)}\left(2 \omega_{1}+\omega_{2}\right) E_{1}^{2} E_{2}+\chi^{(3)}\left(2 \omega_{1}-\omega_{2}\right) E_{1}^{2} E_{2}
\end{aligned}
$$

When the full expressions (eqns. 6 and 7) for $E_{1}$ and $E_{2}$ are substituted into eqn. $(8)$, and the relevant trigonometric identities employed, frequencies of $3 \omega_{1}, 3 \omega_{2}$, $\omega_{1} \pm 2 \omega_{2}$ and $2 \omega_{1} \pm \omega_{2}$ are found.

Similar reasoning indicates that if centrosymmetry is broken, such as when enzymes are transducing energy from an electric field, extra mixing frequencies at the even-order frequencies $2 \omega_{1}, 2 \omega_{2}$ and $\omega_{1} \pm \omega_{2}$ (and $4 \omega_{1}, 4 \omega_{2}, 3 \omega_{1} \pm \omega_{2}$ and $3 \omega_{2} \pm \omega_{1}$, etc.) can be expected to appear due to the inclusion of even-order terms in the expansion of the polarisation equation. However, it should be mentioned that since the coefficients of the harmonic terms in the binomial expansion equivalent to Eqn. (9) are smaller than those of the mixing terms, it is to be expected that the latter (sidebands) will dominate in the observed spectra.

\section{EXPERIMENTAL}

\section{Organism}

Sacharomyces cerevisiae was obtained locally as a freeze-dried powder, and resuspended to a concentration of ca $50 \mathrm{mg}$ dry wt ml $\mathrm{ml}^{-1}$ in a medium consisting of $1 \%(\mathrm{w} / \mathrm{v})$ yeast extract in tap water. All experiments were performed in this medium unless otherwise recorded, within $4 \mathrm{hr}$ after the preparation of the stock cell suspension. The main effects of the change to this medium, which differs from that used previously [1], were that positive rather than negative odd-order harmonics were generated in resting cells, and that the frequency window for their generation was somewhat broadened and the amplitude window slightly narrowed. Variations in the medium components indicated that these effects were due to the greater $\mathrm{Cl}^{-}$ ion concentration in the previous medium (data not shown).

\section{Chemicals}

All of these were obtained from the Sigma Chemical Company or BDH Ltd, Poole, Dorset. 


\section{Apparatus}

The 2-cell, 4-electrode nonlinear dielectric spectrometer which we used was exactly as described previously [1], except that the excitation was provided by means of a Thandor TG501 function generator (RS Components Ltd) (baseline noise level $\cong-45 \mathrm{~dB}$ ) and a Krohn-Hite Model 4400A Ultra-low-noise Generator (whose baseline noise level $\cong-75 \mathrm{~dB}$ ) which were connected to the electrodes via a $10 \mathrm{x}$ mixer. The frequency and amplitude of these signals was checked using a Solartron 1200 Signal Processor (Schlumberger Instruments, Farnborough, Hants) and a Hameg HM 208 Digital Storage Oscilloscope.

The acquisition of the data and its subsequent processing and display was performed using the ILS software (Signal Technology Inc., Goleta, CA), running under control files written in house. In all cases, spectra were collected as follows: the system of interest was pipetted into the electrode cell, the test waveform applied to the (outer) current electrodes, and the data logged, from the (inner) voltage electrodes at a sampling frequency (which was typically at 15 times the frequency of the fundamental) and for a time specified by the operator. Time was specified in terms of a number of blocks, each block consisting of 512 samples. At the end of the time specified, normally equivalent to 30 blocks, the data were windowed and Fourier-transformed as described [1], and stored on the computer's hard disk.

A reference spectrum was acquired using the supernatant, whose conductivity had been adjusted (with distilled water, to compensate for the volume fraction of cells present in the sample; see refs. 20,21) to be identical to that of the sample at the frequency of interest. Two different types of batch file were used, depending upon whether the reference was to be logged using the same set of electrodes or (as was done in the experiments presented herein) a separate matched electrochemical system. In either case, the logging, windowing and Fourier transformation routines were identical, and provided a power spectrum of the "reference" cell, which was also logged on the computer's hard disk. Finally the "sample" power spectrum so obtained was divided by the "reference" power spectrum, and also stored on the disk.

\section{RESULTS}

Figure 1 shows the data from an experiment in which a suspension of $S$. cerevisiae cells were excited with a waveform consisting of sinusoids of $1 \mathrm{~Hz}$ and 15 $\mathrm{Hz}$, each of $\pm 0.6 \mathrm{~V}\left(0.8 \mathrm{~V} \mathrm{~cm}^{-1}\right)$ as measured on the outer electrodes, and displays spectra from (A) the sample, (B) the reference and (C) their difference. The following observations may be made: (i) due to imperfections in the generator and the nonlinearities inherent in electrochemical systems, the applied waveform is not purely sinusoidal but contains power at frequencies other than those set and which, although very small by comparison with the energy in the fundamentals, may yet be observed using a measuring system with the present (16-bit) sensitivity and a logarithmic display; (ii) the power spectra of the "sample" and "reference" cells are markedly different. Inspection of Fig. 1C shows that upon substraction of the 

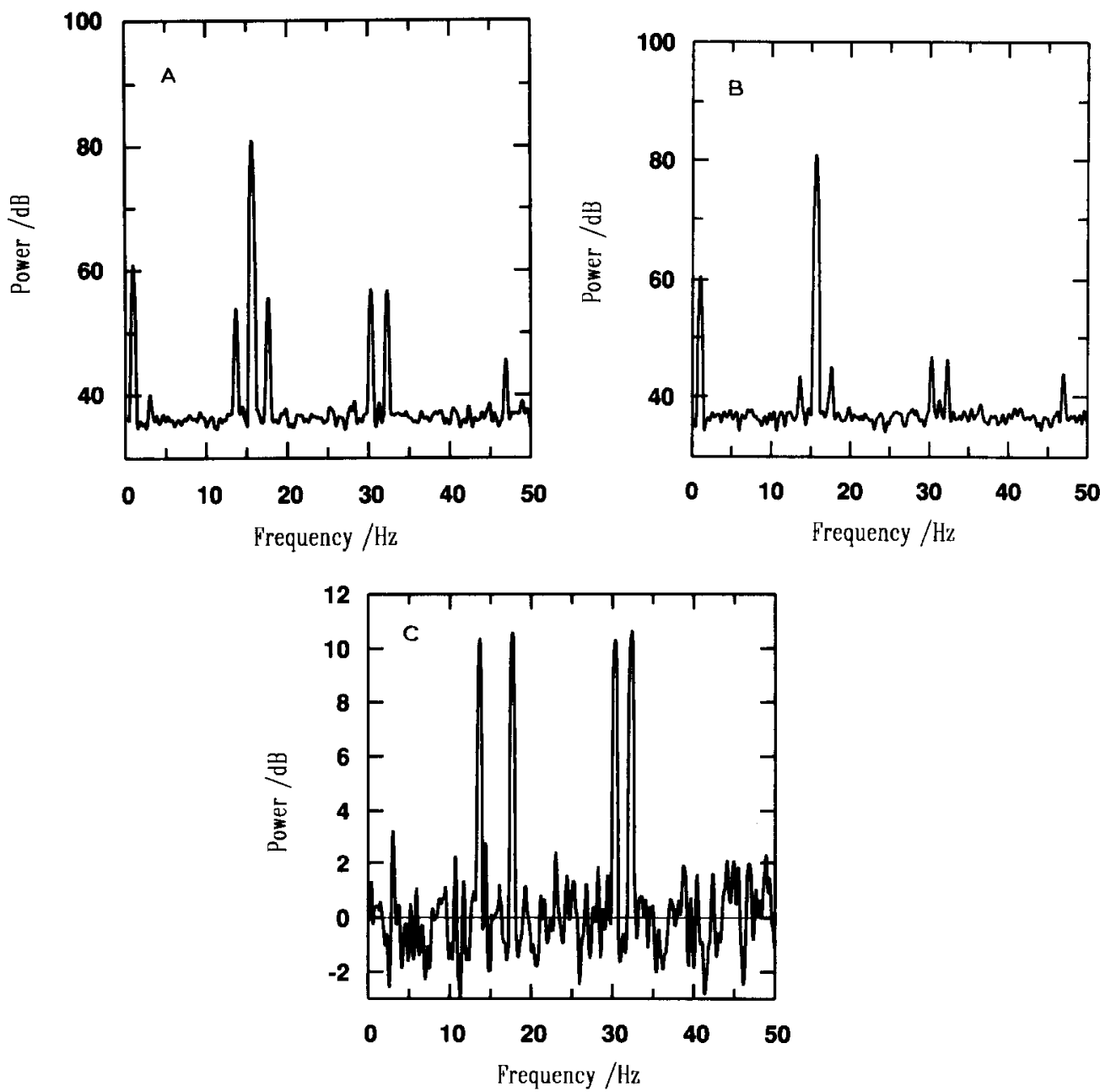

Fig. 1. Nonlinear dielectric properties of $S$. cerevisiae. A suspension of cells $\left(50 \mathrm{mg}\right.$ dry wt $\mathrm{ml}^{-1}$, prepared as described in the Experimental section), was placed in the test cell, and a sample of its supernatant (with its conductivity at $1 \mathrm{kHz}$ adjusted to match that of the cells) was placed in the reference cell. Spectra were obtained using exciting frequencies of $1 \mathrm{~Hz}$ and $15 \mathrm{~Hz}$, each of amplitude (zero-to-peak) of $\pm 0.6 \mathrm{~V}$ (field strength $0.8 \mathrm{~V} \mathrm{~cm}^{-1}$ ), and each spectrum represent the average of 30 blocks. (A) Test cell. (B) Reference cell. (C) Test cell minus reference cell.

reference spectrum from the sample spectrum strong frequency components may be observed at 13,17, 29 and $31 \mathrm{~Hz}$. If the frequencies applied are denoted $f_{1}$ and $f_{2}$, the frequencies dominating the output waveform in the difference spectrum correspond to $2 f_{1}+f_{2}, 2 f_{1}-f_{2}, f_{1}+2 f_{2}$ and $f_{1}-2 f_{2}$. We refer to these frequencies as "beat frequencies" or "sidebands". We would stress that neither of the two waveforms when applied alone was capable of generating harmonics, since individu- 


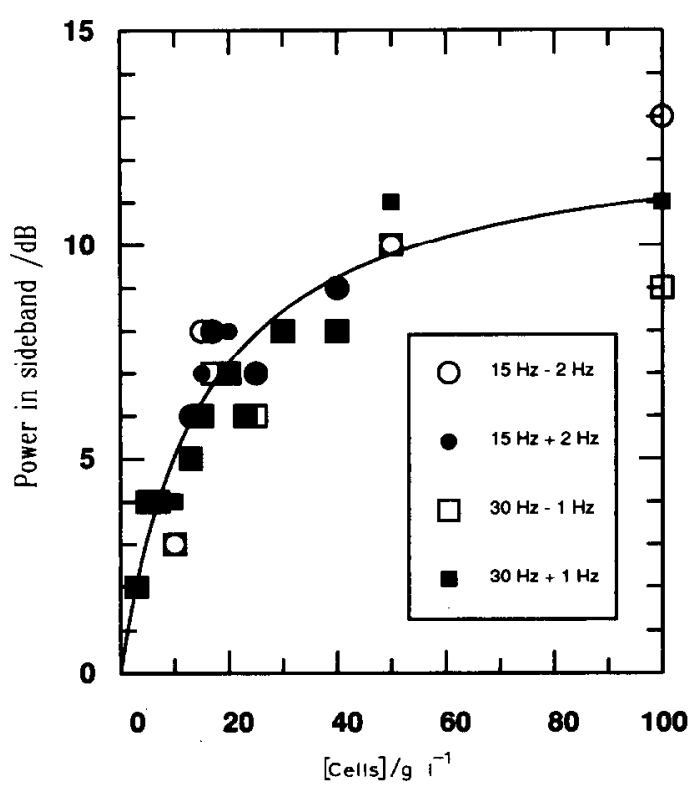

Fig. 2. Effect of cell concentration on the magnitude of the sidebands in the nonlinear dielectric spectrum of $S$. cerevisiae. The experiment was performed as described in the legend to Fig. 1 , and the cell concentration was varied as indicated.

ally the field strengths were below the voltage window previously observed [1]. Thus both frequencies in the waveform must cooperate in the transduction of the exciting electrical energy into beat frequencies.

Control experiments (not shown), similar to those described previously [1], showed that neither matched cell suspensions or supernatants, nor boiled cells, could generate beat frequencies. Figure 2 shows that the generation of sidebands proceeds in a cell-concentration-dependent manner, with a moderately linear portion being followed by a transition towards a plateau region at cell concentrations of approximately $40 \mathrm{~g}^{-1}$, much as in the previous experiments.

The previous experiments [1] showed that the ability to generate harmonics was strongly inhibited by the $\mathrm{H}^{+}$-ATPase inhibitor sodium metavanadate. A vanadate titration of the observable sidebands is given in Fig. 3, where it may be seen that the generation of all sidebands is strongly suppressed by concentrations of vanadate of as little as $50 \mu \mathrm{M}\left(1 \mathrm{nmol} \cdot \mathrm{mg}\right.$ dry weight $\left.{ }^{-1}\right)$, with no preference for any particular sideband.

Since the ability to generate a third harmonic when resting cell suspensions were excited with a "pure" sinusoid depended strongly upon the frequency of the exciting signal [1], it was of interest to sec how (or even whether) the ability to generate sidebands varied when one exciting frequency was kept constant and the other varied. As in Fig. 1, the field strengths applied with each frequency were \pm 0.8 $\mathrm{V} \mathrm{cm}^{-1}$, and the constant frequency was $15 \mathrm{~Hz}$, the second frequency being varied 


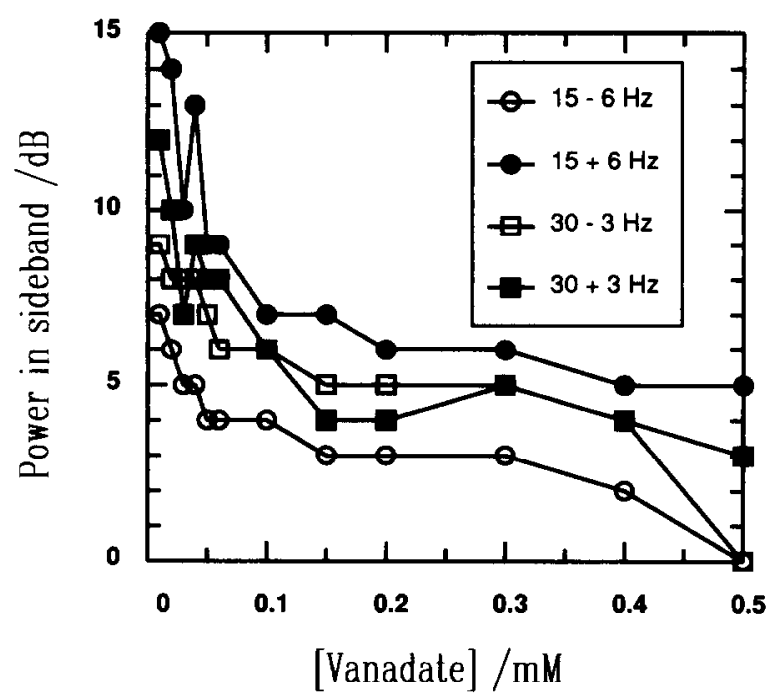

Fig. 3. Sensitivity to vanadate of the sidebands in the nonlinear dielectric spectrum of $S$. cerevisiae. Experiments were performed as described in the legend to Fig. 1, except that the frequencies of excitation were $3 \mathrm{~Hz}$ and $15 \mathrm{~Hz}$. Sodium metavanadate was added to the concentrations indicated.

as indicated on the abscissa of Fig. 4. It may be observed (Fig. 4) that there is again a fairly clearly defined frequency window for the generation of beat frequencies, with no clear discrimination between individual beat frequencies when the variable frequency is high but with a significant emphasis on the $15 \pm 2 f_{2}$ sidebands when the variable frequency is low (relative to $15 \mathrm{~Hz}$ ). There is also a possible suggestion of further stimulating processes centred at frequencies below $1 \mathrm{~Hz}$, but this was not

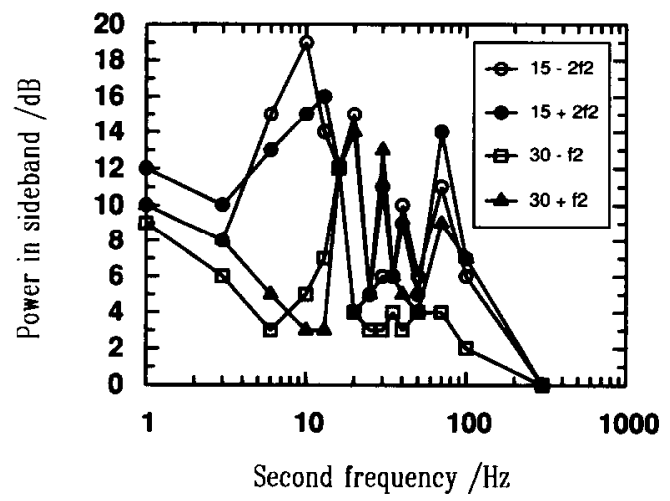

Fig. 4. Effect of exciting frequencies on the magnitude of the sidebands of the nonlinear dielectric spectra of $S$. cerevisiae. The experiment was performed as described in the legend to Fig. 1 , except that one exciting frequency was maintained at $15 \mathrm{~Hz}$ whilst the second exciting frequency was varied as indicated. 


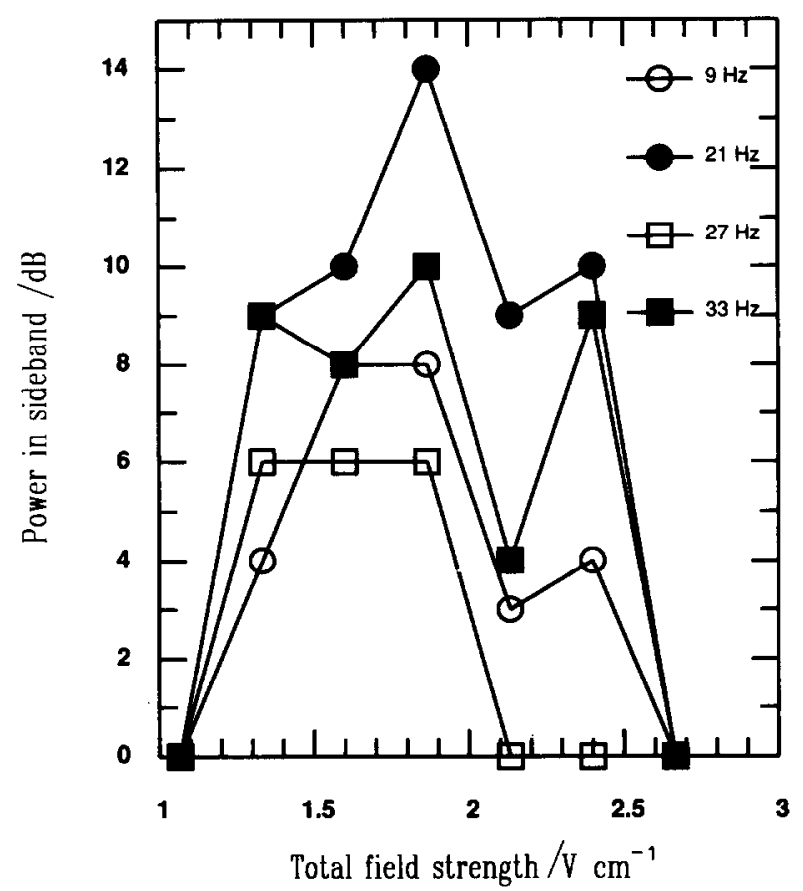

Fig. 5. Effect of exciting field strength on the magnitude of the sidebands of the nonlinear dielectric spectra of $S$. cerevisiae. The experiment was performed as described in the legend to Fig. 3, except that whilst the amplitude of each exciting frequency was equal, the overall amplitude was varied as indicated.

investigated further, due to the well-known instability of function generators at sub-Hz frequencies. It is worth pointing out again that the "variable" frequency could be well outside the frequency window observed [1] when only single sinusoids were applied. At the amplitudes of the exciting fields employed, sidebands could be observed provided that the arithmetic mean of the 2 frequencies lay within or close to the frequency window observed [1] for pure sinusoidal excitation.

As well as a frequency window, there was a marked amplitude window. To illustrate this, an experiment similar to those displayed in Figs. 1 to 4 was performed, with excitation at $3 \mathrm{~Hz}$ and $15 \mathrm{~Hz}$, each applied sinusoid being of equal field strength (peak-to-peak), as judged by measurements on the outer electrodes, save that the total field strength was varied. The data are illustrated in Fig. 5, where it may be observed that the "overall" field amplitude for the generation of sidebands manifests a window with a peak at approximately $2 \mathrm{~V} \mathrm{~cm}^{-1}$, much as in the experiments [1] with pure sinusoidal excitation. It is not wholly obvious why there is a fall-off in harmonic or sideband structure at the higher voltages, and it is worth mentioning that at voltages in excess of some $\pm 2 \mathrm{~V}$ (zero-to-peak; field strengths of $\pm 2.7 \mathrm{~V} \mathrm{~cm}^{-1}$ ) electrodeposition processes tended to obscure the ability to observe clean nonlinear dielectric spectra. 


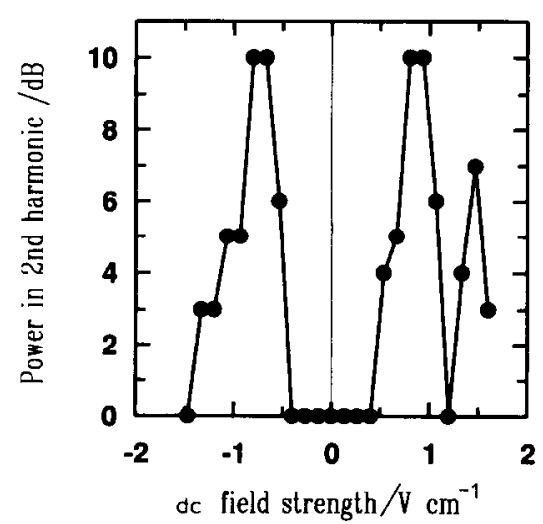

Fig. 6. Effect of a dc electrical field on the generation of harmonics by suspensions of $S$. cerevisiae. Measurements were performed as described in the legend to Fig. 1, except that the single exciting frequency was $15 \mathrm{~Hz}$ at $\pm 0.8 \mathrm{~V} \mathrm{~cm}^{-1}$ and the dc field was varied as indicated.

It is possible to consider a dc field as a field of frequency $0 \mathrm{~Hz}$, such that its additional imposition on top of a single-frequency excitation might produce sidebands as predicted above. Furthermore, the mixing frequencies are then expected to occur at $2 f_{1}+f_{2}, 2 f_{1}-f_{2}, f_{1}+2 f_{2}$ and $f_{1}-2 f_{2}$. These frequencies coincide at $2 f_{2}$, when $f_{1}=0 \mathrm{~Hz}$ and where $f_{2}$ is the frequency of the imposed sinusoid. To assess this possibility, we carried out an experiment in which we applied a single sinusoid of $15 \mathrm{~Hz}$ (whose amplitude $\left(0.8 \mathrm{~V} \mathrm{~cm}^{-1}\right)$ was below the voltage window). The idea was that, since the imposition of a dc field in the presence of a pure sinusoid constitutes a special case of 2-frequency mixing, increasing the value of a dc field should serve to bring the overall field strength within the amplitude window. As shown in Fig. 6, this suggestion was indeed borne out: the second harmonic increases as the (modulus of the) dc field is raised from zero, passing, as usual, through a maximum to give, in this case, two overall "amplitude windows" on either side of the origin. Indeed, in our previous work [1], we had noted that if the measurements were carried out with a sinusoid of the optimum amplitude but in the presence of an additional electrostatic (dc) field, the magnitude of the 3rd harmonic was strongly decreased, disappearing completely when the dc field exceeded $\pm 0.4 \mathrm{~V}$ $\mathrm{cm}^{-1}$ (and the exciting ac field was $2.0 \mathrm{~V} \mathrm{~cm}{ }^{-1}$ ). Thus it would appear that the effect of a dc field in the earlier case is simply explained as its ability to take the overall (combined) amplitude of the exciting field outside the amplitude window.

To explore this point further, we carried out a 2-frequency excitation experiment in which an additional dc field was imposed. The dc component imposes a rectification on the overall exciting signal, introducing even-order terms into the expansion of the polarisation equation. As the dc field increases (from zero), the even-order sidebands $f_{1} \pm f_{2}$ may be expected to increase (from zero) at the expense of the odd-order terms $f_{1} \pm 2 f_{2}$ and $2 f_{2} \pm f_{1}$. This effect may be seen clearly in Fig. 7. As the dc component continues to increase, the upper limit of the amplitude window is reached and all signals disappear (Fig. 7.). 


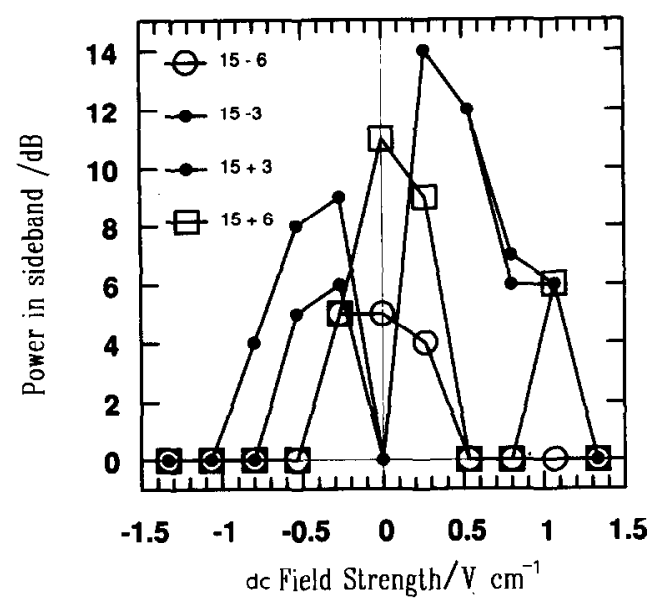

Fig. 7. Effect of a dc electrical field on the generation of sidebands by suspensions of $S$. cerevisiae. Measurements were performed as decribed in the legend to Fig. 3, except that the amplitude of each sinusoidal excitation was $\pm 0.5 \mathrm{~V}$ (zero-to-peak) and a dc field was additionally imposed as indicated.

As discussed previously [1], the addition of a glycolytic substrate to a resting cell suspension activated the $\mathrm{H}^{+}$-ATPase and led to the generation of even-order (second and fourth) harmonics in the nonlinear dielectric spectrum, as depicted in Fig. 8. As discussed above, this corresponds to the presence of second- and fourth-order terms in the polarisation expansion. In the experiment displayed, the

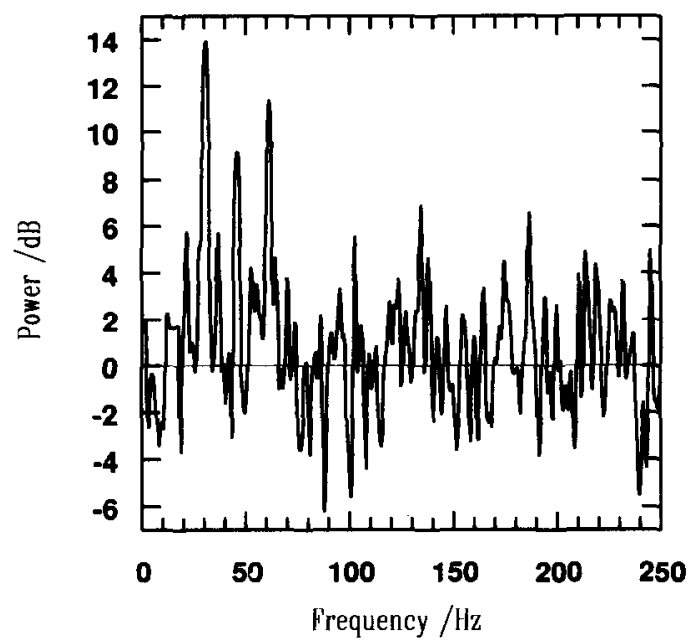

Fig. 8.The ability of glycolysing cells of $S$. cerevisiae to generate even-order harmonics. The experiment was performed as described in the Materials and Methods section, with an exciting frequency of $15 \mathrm{~Hz}$ $\left( \pm 1.6 \mathrm{~V} \mathrm{~cm}^{-1}\right)$. Glucose was added to a final concentration of $500 \mathrm{mM}$ and the temperature was $40^{\circ} \mathrm{C}$. 


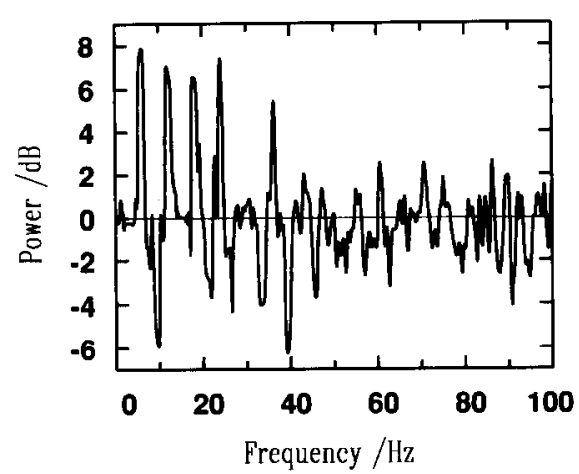

Fig. 9. The ability of glycolysing cells of $S$. cerevisiae to generate even-order sidebands. The exciting frequencies were $3 \mathrm{~Hz}$ and $15 \mathrm{~Hz}$, each with an amplitude $0.8 \mathrm{~V} \mathrm{~cm}^{-1}$ (zero-to-peak).

ATPase was probably not turning over at its maximum rate, so that the spectrum also contained a (smaller) third harmonic. Therefore, we next investigated the theoretically predicted generation of second- and fourth-order sidebands when the same cell suspension was subjected to two-frequency excitation (Fig. 9). Positive, sccond- and fourth-order sidebands $\left(f_{1} \pm f_{2}, f_{1} \pm 3 f_{2}\right)$ are generated, along with smaller negative ones of third-order $\left(f_{1} \pm 2 f_{2}\right)$. Thus, the presence of even-order sidebands is diagnostic, and provides a sensitive means of detection, of ongoing metabolism in biological cell suspensions.

\section{DISCUSSION}

There is increasing interest in the nonlinear properties of both biological and abiological dynamical systems. Leaving aside the many and ongoing studies of non-linear optical systems, in this context, one may mention nerve axons [22-25], ferroelectric polymers [26-32] (and these two categories may be related [33,34]), and the phospholipid bilayer-based oscillatory electrochemical system described by Yoshikawa and coworkers [35], each of which has been show to produce harmonics when excited with a single sinusoid. It is also to be expected that the excitable biomimetic phospholipid membrane system of Toko and colleagues [36-38] would behave similarly. Moussavi et al. [39] describe a recent implementation of a system for determining the generation of harmonics by purely electrochemical systems.

We recently described [1] the generation of nonlinear dielectricity by cell suspensions of the yeast Saccharomyces cerevisiae, within fairly narrow voltage and frequency windows. This was carried out by providing excitation with a single sinusoid whilst measuring the response at a range of frequencies by Fourier transformation of the resultant voltage signal. As well as their ability to generate harmonics, nonlinear systems have the property that they if they are excited by 2 frequencies simultaneously they can generate sums and differences of integral multiples of the exciting frequencies, a technique exploited for instance in the study 
of polymer dielectrics by Ikeda et al. [29]. We have now extended our own work to the exploitation of 2 -frequency excitation for the study of the nonlinear dielectric properties of biological (and other) systems.

We showed (Fig. 1) that the excitation of resting suspensions of yeast cells with frequencies of $1 \mathrm{~Hz}$ and $15 \mathrm{~Hz}$ (the latter being close to the optimum for single-frequency excitation [1]), with amplitudes $\left( \pm 0.8 \mathrm{~V} \mathrm{~cm}^{-1}\right)$ which on their own would not cause the generation of harmonics, led to the production of sidebands at frequencies corresponding to $f_{1} \pm 2 f_{2}$ and $f_{2} \pm 2 f_{1}$. That the individual exciting frequencies could not alone cause the generation of harmonics indicates clearly that both frequencies were cooperating in the generation of the nonlinear dielectricity, i.e. that they were not acting independently in the way which would be expected for a classical dielectric relaxation conforming, via the superposition principle, to the Debye [40] or Cole-Cole [41] type of analysis [42-52]. The use of 2-frequency excitation therefore provides a particularly clcar-cut and powerful means for demonstrating the cooperation between individual frequency components in the generation of nonlinear dielectricity.

As regards the reproducibility of these experiments, the data displayed could be reproduced within a few $\mathrm{dB}$ between batches of cells, and somewhat better within an individual batch. However, there were some day-to-day variations in the optimal frequency and amplitude of excitation, as may be judged for instance by comparing the data in Figs. 1 and 4.

To check that the source of the nonlinear dielectricity in these 2-frequency-excitation experiments was indeed the membrane $\mathrm{H}^{+}$-ATPase in this organism, we carried out a titration with sodium metavanadate, a well-known inhibitor which acts as a transition-state analogue, trapping the enzyme in its $E_{2}$ form [53-55]. It was found [Fig. 3] that vanadate was indeed a potent inhibitor of the ability of the cells to generate sidebands, with no particular discrimination between individual sidebands. This is exactly the behaviour one might expect of a tight-binding inhibitor.

When one frequency was held at $15 \mathrm{~Hz}$ and the other allowed to vary (each waveform having an applied amplitude of $\pm 0.8 \mathrm{~V} \mathrm{~cm}^{-1}$ ), we again observed a frequency window for the second frequency (Fig. 4). With single-frequency excitation, we could observe only the 3rd harmonic in resting cell suspensions [1]. However, because the 2-frequency excitation generated at least 4 sidebands, it was possible to distinguish each of these as the second excitation frequency was varied. We observed (Fig. 4) that "co-frequencies" of excitation lower than the "main" 15 $\mathrm{Hz}$ excitation favoured the generation of sidebands at $15 \pm 2 f_{2}$, even above the equivalent 3rd-harmonic generated when $f_{1}=f_{2}=15 \mathrm{~Hz}$, whilst there was no obvious discrimination amongst the different sidebands when $f_{2}$ was greater than $15 \mathrm{~Hz}$. Because the ability of exogenous electrical fields to induce electroconformational coupling depends upon all of the individual rate constants in the "target" enzyme, it is to be assumed that the overall 2-frequency spectra reflect these transitions in some way. Whilst Astumian and Robertson $[9,56]$ have discussed the generation of nonlinear dielectricity by membranous enzymes whose rate constants are known, the inverse problem has not yet been solved, so that it is not presently 
possible to deconvolve our nonlinear dielectric spectra so as to obtain the individual rate constants of the target enzyme(s).

As well as a 2-frequency window, there was an overall amplitude window when resting cell suspensions of $S$. cerevisiae were excited with frequencies of $15 \mathrm{~Hz}$ and 3 $\mathrm{Hz}$ (Fig. 5). The overall amplitude window (ca. $\pm 1-2.7 \mathrm{~V} \mathrm{~cm}^{-1}$, zero-to-peak) as measured with the outer electrodes was essentially similar to that observed with single-frequency excitation [1].

In our previous work, we found that the imposition of a dc field, on top of an ac field of optimum frequency and amplitude, strongly inhibited the ability of yeast cells to generate nonlinear dielectricity [1]. As described in the Theory section, a "mixed" excitation consitutes a special case of 2-frequency excitation, with the dc field being equivalent to an exciting frequency of zero $\mathrm{Hz}$. Given the amplitude window observed above, it was predicted that the imposition of a dc field on top of a single-frequency excitation whose magnitude was suboptimal would allow us to observe second harmonics $\left(f_{1}+2 f_{2} ; f_{1}=0 \mathrm{~Hz}\right)$ from resting cell suspensions within a suitable amplitude window. This is exactly what was found (Fig. 6).

To extend this analysis, we also carried out an experiment in which resting cells were subjected to 2-frequency excitation in the usual way (Fig. 7), generating, as usual, sidebands corresponding to $f_{1} \pm 2 f_{2}$. The cells were then additionally subjected to a dc field. As this was increased (in either polarity) the odd-order sidebands decreased and even-order sidebands were generated. Ultimately, the level of the dc ficld caused the overall excitation to exceed the amplitude window, and no sidebands were generated. This behaviour is also exactly that to be expected from the theory described in the Theory section.

When we studied the cell-concentration-dependence of the ability of suspensions of $S$. cerevisiae to generate sidebands, we found that their magnitude was linear only up to cell concentrations of $30 \mathrm{~g} \mathrm{l}^{-1}$ or so, whereupon there was a transition to a plateau region in which increases in cell concentration did not lead to increases in the magnitude of the sidebands (Fig. 2). Similar behaviour was observed in the experiments with single-frequency excitation [1]. It would appear that high cell concentrations modulate the local field intensity such that it is decreased away from the normal, optimum voltage window.

In the previous work [1], and in the present experiments, we have described the field strengths applied in terms of those measured on (the electrode side of) the outer electrodes. The peak excitations were found, on this basis (at a frequency of $15-20 \mathrm{~Hz}$ ), at voltages of some $\pm 1.5 \mathrm{~V}$ (zero-to-peak), corresponding in our electrode geometry [1] to field strengths of some $\pm 2 \mathrm{~V} \mathrm{~cm}^{-1}$. We have now measured the field strengths as they fall across the inner electrodes, and find that for ostensibly applied fields of $\pm 2 \mathrm{~V} \mathrm{~cm}^{-1}$, as judged on the electrode side of the outer electrodes, the outer electrode impedances, and the $\mathrm{iR}$ drop in the medium, are such that the local electric field across the inner electrodes is only some $153 \mathrm{mV}$ (zero-to-peak) $\mathrm{cm}^{-1}$. Thus the effective field strengths in these experiments, and the effective Langevin factor [1], are even lower, by a factor of approximately 13, than those calculated on the basis of the apparently applied field. 
Finally, we wish to discuss in more detail than was possible before: the resolution of our measuring system, the efficiency of coupling between the exciting electric fields and the target enzyme, and the relationship(s) between the nonlinear dielectric behaviour observed and the catalytic activity of the target enzyme.

\section{Measurement limitations}

As may be observed in the nonlinear dielectric spectra displayed herein, we typically observe a frequency independent "baseline" noise at a level some $45 \mathrm{~dB}$ below the level of the exciting waveform. The variance of the noise in this baseline, and in the ability to discrimate different voltages and frequencies, may be improved by signal averaging [1], but the absolute baseline level is fixed by the noise level generated by the Thandor generator. When only the low-noise Krohn-Hite generator was used to provide single-frequency excitation, quantisation inherent in the A/D process becomes significant.

The signal power to quantisation noise power ratio is given by [57] as $S / n=3 r^{2} / 2$

where $r$ is the ratio of total signal excursion to quantisation step. This predicts an decrease of $6 \mathrm{~dB}$ in the noise baseline for each additional bit (sensu binary digit) of resolution. Table 1 shows the relationship between the measured baseline noise (with respect to a $\pm 1.2 \mathrm{~V}$ sinusoidal signal) and the bit resolution of the $\mathrm{A} / \mathrm{D}$ converter. The same formula also predicts the correct baseline noise for the 12 bit digitisation.

It can be seen from Table 1 that the expected $12 \mathrm{~dB}$ decrease in noise level for an increment of 2 bits resolution is reflected between 12 and 14 bits, showing that quantisation is the predominant noise source in these readings. However, if the resolution is increased by a further 2 bits, to 16 bits, only a $5 \mathrm{~dB}$ improvement is produced. This suggests that the baseline is now approaching that of the noise produced by the generator, and significant improvements cannot be obtained by further increasing the resolution of the A/D process.

At 12 bits, all but large harmonics begin to be lost in the noise. At 14 bits smaller features are present, but still not to the desired clarity, so it is seen that 16 bits is the minimum resolution which can produce satisfactory definition of the smallest

\section{TABLE 1}

The baseline noise in the present system as a function of the resolution of the Analog-to-Digital converter used. A cell suspension was prepared and analysed using the Krohn-Hite generator as described in the Experimental section, save that the resolution of the A-D process was artificially degraded from the normal 16 bits prior to Fourier transformation

\begin{tabular}{ll}
\hline Resolution/bits & Noisc baseline relative to fundamental/dB \\
\hline 12 & -60 \\
14 & -70 \\
16 & -75 \\
\hline
\end{tabular}


details present in the signal. Consequently, 16 bits is seen to be the optimum resolution for this system.

The $\mathrm{H}^{+}-$ATPase in the plasma membrane of $S$. cerevisiae

The $\mathrm{H}^{+}$-ATPase in the plasma membrane of $S$. cerevisiae is in many ways a complex enzyme, whose activity depends not only on the concentration of its substrates and products, but on the nature of its associated lipids and whether it is incorporated in a vesicle which is ion-tight or leaky. Our interest is focussed upon its kinetic parameters in vivo. It is known that this enzyme is essential for the growth of the cell [58], and under acidic conditions has a flux-control coefficient (see ref. 59) for growth of 1 [60]. It has a major subunit with a molecular weight of almost exactly 100,000 [61-63], and would appear to be a classical $\mathrm{E}_{1} \mathrm{E}_{2}$ - or P-ATPase, with a stoichiometry of $1 \mathrm{H}^{+}$/ATP hydrolysed under optimally coupled conditions [64], and with the (most) rate-limiting step being the conversion of $E_{2}$ to $E_{1}[15]$. The gene ( $P M A 1)$ coding for the main enzyme has been cloned and sequenced $[58,65]$, and is being subjected to site-directed mutagenesis [66,67]. Interestingly, expression of the yeast $\mathrm{H}^{+}$-ATPase in mouse fibroblasts produces a tumorigenic type of transformation [68]. Recent evidence suggests that the enzyme may also contain a small, proteolipid subunit [69], and may exhibit a close functional linkage to the major $\mathrm{K}^{+}$channel in the plasma membrane [70].

In normal yeast cells, the enzyme is present in amounts typically corresponding to $10-15 \%$ of the membrane protein of the cell [14] and has an apparent $k_{\text {cat }}$ (based on a molecular weight of $100 \mathrm{kD}$ ) when embedded in the native membrane of 30-45 $s^{-1}[71]$ or $36 s^{-1}$ [14]. There is genetic and other evidence, however, that the active enzyme in situ is dimeric [72,73], so that this value must then be divided by 2 . When this is done, and taking into account the decrease in $k_{\text {cat }}$ when the enzyme is in a more-or-less native, ion-tight membrane, all values of $k_{\text {cat }}$ fall in the decade 10-100 $\mathrm{s}^{-1}$ with perhaps the most representative being some $10-25 \mathrm{~s}^{-1}$. Whilst we could not directly measure the turnover number of the enzyme in vivo (and for this even saturation transfer NMR requires a knowledge of the number of ATPases present [74], since it measures only the total flux), it would appear that the optimum frequency of excitation for the generation of nonlinear dielectricity matches rather exactly the maximum turnover number of the target enzyme itself. If this were indeed he case, nonlinear dielectric spectroscopy would for the first time permit the registration of intrinsic enzyme catalytic properties in situ, without the necessity to know the number of active enzymes present.

\section{Efficiency of energy transduction}

It is of obvious interest to derive an estimate of the proportion of the electrical energy which is being utilised by the $\mathrm{H}^{+}$-ATPase, if the generation of harmonics is representative of the normal mode of energy transduction in the enzyme.

The electrical power provided by the applied field is given by

$E_{\mathrm{rms}}^{2} G$ 
where $E_{\mathrm{rms}}$ is the root mean square value of the voltage and $G$ is the conductance of the suspension. For an input of $1.2 \mathrm{~V}$ amplitude, the rms value across the inner electrodes is $80 \mathrm{mV}$. The conductance of the suspension between the inner electrodes was measured to be $0.9 \mathrm{mS}$. These figures give a value for the electrical power of $6 \mu \mathrm{W}$.

A yeast suspension contains approx. $10^{6}$ cells $(\mathrm{mg} / \mathrm{ml})^{-1}[75]$, so the suspension used above contains approx. $5 \times 10^{7}$ cells $/ \mathrm{ml}$. If each cell contains $2000 \mathrm{H}^{+}$-ATPase enzymes, the suspension contains approx. $10^{11}$ enzymes $/ \mathrm{ml}$ or some $2 \times 10^{-13}$ moles of enzyme $/ \mathrm{ml}$.

In the process of its turnover, each mole may be taken to transduce some $42 \mathrm{~kJ}$ [76], so $1 \mathrm{ml}$ of yeast suspension at a concentration of $50 \mathrm{mg} / \mathrm{ml}$ generates approx. $10^{-8} \mathrm{~J} /$ turnover. At an optimum turnover rate of $20 \mathrm{~Hz}$, this corresponds to a power output of $0.2 \mu \mathrm{W}$, assuming all enzymes are turning over.

Whilst the present calculation is necessarily crude, the efficiency of energy usage by the enzymes based on the above analysis $\cong 3 \%$. This is not dissimilar to the figures suggested by Roberston et al. [77] from electroconformational coupling considerations [78-80].

\section{ACKNOWLEDGEMENTS}

We are indebted to the Wolfson Foundation for financial support of this work. D.B.K. wishes to acknowledge with pleasure many stimulating discussions of these and other topics with Drs. Dean Astumian, Baldwin Robertson, Bob Todd and Hans Westerhoff.

\section{REFERENCES}

1 A.M. Woodward and D.B. Kell, Bioelectrochem. Bioenerg., 24 (1990) 83.

2 A.R. Fersht, Enzyme Structure and Mechanism, Freeman, San Francisco, 1977.

3 G.R. Welch and D.R. Kell, in G.R. Welch (Ed.), The Fluctuating Enzyme, Wiley, New York, 1986, p. 451.

4 D.B. Kell, Univ. Wales Rev. Sci. Technol., 1 (1988) 64.

5 D.B. Kell, ISI Atlas Sci., Biochemistry, 1 (1988) 25.

6 D.B. Kell, in H. Fröhlich (Ed.), Biological Coherence and Response to External Stimuli, Springer, Heidelberg, 1988, p. 233.

7 D.B. Kell, R.D. Astumian and H.V. Westerhoff, Ferroelectrics, 86 (1988) 59.

8 H.V. Westerhoff, R.D. Astumian and D.B. Kell, Ferroelectrics, 86 (1988) 79.

9 R.D. Astumian and B. Robertson, J. Chem. Phys., 91 (1989) 4891.

10 A.K. Jonscher, Dielectric Relaxation in Solids, Chelsea Dielectrics Press, London, 1983.

11 L. Dissado and R.M. Hill, Proc. R. Soc. Ser. A, 390 (1983) 131.

12 R.M. Hill and A.K. Jonscher, Contemp. Phys., 24 (1983) 75.

13 R.G. Palmer, D.L. Stein, E. Abrahams and P.W. Anderson, Phys. Rev. Lett., 53 (1984) 958.

14 A. Wach, J. Ahlers and P. Gräber, Eur. J. Biochem., 189 (1990) 675.

15 A. Goffeau and N.M. Green, in C.A. Pasternak (Ed.), Monovalent Cations in Biological Systems, CRC Press, Boca Raton, FL, 1990, pp. 155-169.

16 Y.R. Shen, Nature, 337 (1989) 519.

17 G.C. Baldwin, An Introduction to Nonlinear Optics, Plenum, New York, 1969. 
18 D.J. Williams, Angew. Chem. Int. Ed. Engl., 23 (1984) 690.

19 D.B. Kell and A.M. Woodward, in H. Westerhoff (Ed.), Proc. 4th BTK Symposium, Amsterdam, Intercept Press, London, 1991.

20 C.M. Harris and D.B. Kell, Bioelectrochem. Bioenerg., 11 (1983) 15.

21 R.W. Lovitt, R.P. Walter, J.G. Morris and D.B. Kell, Appl. Microbiol. Biotechnol., 23 (1986) 168.

22 L.J. DeFelice, W.J. Adelman Jr., D.E. Clapham and A. Mauro, in W.J. Adelman Jr. and D.E Goldman (Eds), The Biophysical Approach to Excitable Membranes, Plenum, New York, 1981, p. 37

23 S. Miyamoto and H.M. Fishman, IEEE Trans. Biomed. Eng., BME-33 (1986) 644.

24 H.M. Fishman, H.R. Leuchtag and L.E. Moore, Biophys. J., 43 (1983) 293.

25 R. FitzHugh, Biophys. J., 42 (1983) 11.

26 T. Furukawa, K. Nakajima, T. Koizumi and M. Date, Jap. J. Appl. Phys., 26 (1987) 1039.

27 T. Furukawa, M. Tada, K. Nakajima and I. Seo, Jap. J. Appl. Phys., 27 (1988) 200.

28 S. Ikeda, H. Suzuki, K. Koyama and Y. Wada, Polym. J., 19 (1987) 681.

29 S. Ikeda, H. Kominami, K. Koyama and Y. Wada, J. Appl. Phys., 62 (1987) 3339.

30 M. Date, E. Fukada and J.H. Wendorff, IEEE Trans El. Insul., EI-24 (1989) 457.

31 T. Furukawa, Phase Transitions, 18 (1989) 143.

32 T. Furukawa, IEEE Trans El. Insul., EI-24 (1989) 375.

33 H.R. Leuchtag, J. Theor. Biol., 127 (1987) 321.

34 H.R. Leuchtag, J. Theor. Biol., 127 (1987) 341.

35 S. Nakata, K. Yoshikawa, M. Shoji, H. Kawakami and T. Ishii, Biophys. Chem., 34 (1989) 201.

36 S. Iiyama, K. Toko and K. Yamafuji, Biophys. Chem., 28 (1987) 129.

37 K. Hayashi, K. Yamafuji, K. Toko, N. Ozaki and T. Yoshida, Sensors Actuators, 16 (1989) 25.

38 K. Hayashi, M. Yamanaka, K. Toko and K. Yamafuji, Sensors Actuators B, 2 (1990) 205.

39 M. Moussavi, H.H. Sun, H.P. Schwan and A. Richter, Ann. Biomed. Eng., 18 (1990) 505.

40 P. Debye, Polar Molecules, Dover, New York, 1929.

41 K.S. Cole and R.H. Cole, J. Chem. Phys., 9 (1941) 1981.

42 H.P. Schwan, Adv. Biol. Med. Phys., 5 (1957) 147.

43 E.H. Grant, G.P. South and R.J. Sheppard, Dielectric Properties of Biological Molecules in Solution, Oxford University Press, Oxford, 1978.

44 O. Schanne and E.R.P. Ceretti, Impedance Measurements in Biological Cells, Wiley, Chichester, 1978.

45 R. Pethig, Dielectric and Electronic Properties of Biological Materials, Wiley, Chichester, 1979.

46 D.B. Kell and C.M. Harris, J. Bioelectricity, 4 (1985) 317.

47 K.R. Foster and H.P. Schwan in C. Polk and E. Postow (Eds.), CRC Handbook of Biological Effects of Electromagnetic Fields, CRC Press, Boca Raton, FL, 1986, p. 27.

48 D.B. Kell, in A.P.F. Turner, I. Karube and G.S. Wilson (Eds.), Biosensors: Fundamentals and Applications. Oxford University Press, Oxford, 1987, p. 428.

49 R. Pethig and D.B. Kell, Phys. Med. Biol., 32 (1987) 933.

50 K.R. Foster and H.P. Schwan, CRC Crit. Rev. Biomed. Eng., 17 (1989) 25.

51 C.L. Davey and D.B. Kell, in: R. Paris (Ed.), Electric Field Phenomena in Biological Systems. IOP Short Meetings Series 21, Institute of Physics, London, 1989, p. 51.

52 S. Takashima, Electrical Properties Properties of Biopolymers and membranes, Adam Hilger, Bristol, 1989.

53 B.J. Bowman and C.W. Slayman, J. Biol. Chem., 254 (1979) 2928.

54 R.L. Smith, K. Zinn and L.C. Cantley, J. Biol. Chem., 255 (1980) 9852.

55 R.K. Nakamoto and C.W. Slayman, J. Bioenerg. Biomembr., 21 (1989) 621.

56 B. Robertson and R.D. Astumian, Biophys. J., 57 (1990) 689.

57 W.R. Bennett, Bell. Syst. Tech. J., 27 (1948) 447.

58 R. Serrano, M.C. Kielland-Brandt and G.R. Fink, Nature, 319 (1986) 689.

59 D.B. Kell, K. van Dam and H.V. Westerhoff, Symp. Soc. Gen. Microbiol., 44 (1989) 61.

60 F. Portillo and R. Serrano, Eur. J. Biochem., 186 (1989) 501.

61 A. Goffeau and C.W. Slayman, Biochim. Biophys. Acta, 639 (1981) 197.

62 R. Serrano, Curr. Top. Cell. Reg., 23 (1984) 87.

63 N. Nelson and L. Taiz, Trends Biochem. Sci., 14 (1989) 113. 
64 D.S. Perlin, M.J.D. San Francisco, C.W. Slayman and B.P. Rosen, Arch. Biochem. Biophys., 248 (1986) 53.

65 K.P. Holzer and G.G. Hammes, J. Biol. Chem., 264 (1989) 14389.

66 F. Portillo and R. Serrano, EMBO J., 7 (1988) 1793.

67 R. Serrano and F. Portillo, Biochim. Biophys. Acta, 1018 (1990) 195.

68 R. Perona, F. Portillo, F. Giraldez and R. Serrano, Mol. Cell Biol., 10 (1990) 4110.

69 A. Goffeau, A. Coddington and A. Schlesser, in A. Nasim, P. Young and B.F. Johnson (Eds.), Molecular Biology of the Fission Yeast, Academic Press, London, 1990, pp. 397-429.

70 J.A. Ramirez, V. Vacata, J.H. McCusker, J.E. Haber, R.K. Mortimer, W.G. Owen and H. Lecar, Proc. Natl. Acad. Sci., 86 (1989) 7866.

71 J.G. Koland and G.G. Hammes, J. Biol. Chem., 261 (1986) 5936.

72 B.J. Bowman, C.J. Berenski and C.Y. Yung, J. Biol. Chem., 15 (1985) 8726.

73 J.H. McCusker, D.S. Perlin and J.E. Haber, Mol. Cell Biol., 7 (1987) 4082.

74 K. Brindle, P. Braddock and S. Fulton, Biochemistry, 29 (1990) 3295.

75 D.B. Kell, G.H. Markx, C.L. Davey and R.W. Todd, Trends Anal. Chem., 9 (1990) 190.

76 R.K. Thauer, K. Jungermann and K. Decker, Bacteriol. Rev., 41 (1977) 100.

77 B. Robertson, R.D. Astumian and T.Y. Tsong, in M.J. Allen, S.F. Cleary and F.M. Hawkridge (Eds.), Charge and Field Effects in Biosystems 2, Plenum press, New York, 1989, pp. 191-209.

78 T.Y. Tsong and R.D. Astumian, Progr. Biophys. Mol. Biol., 50 (1987) 1.

79 T.Y. Tsong and R.D. Astumian, Annu. Rev. Physiol., 50 (1988) 273.

80 H.V. Westerhoff, D.B. Kell and R.D. Astumian, J. Electrostatics, 21 (1988) 257. 